\title{
HIPERPLASIA ENDOMETRIAL PSEUDO-PLACENTACIONAL EN LA PERRA: UNA REVISIÓN SUCINTA
}

\author{
Endometrial hyperplasia pseudo-placental hyperplasia in the bitch: \\ a succinct review
}

\author{
Alfonso Eduardo Sánchez Riquelme \\ E-mail: profesanchez@gmail.com
}

${ }^{1}$ Facultad de Medicina Veterinaria y Agronomía, Escuela de Medicina Veterinaria, Universidad de Las Américas, Viña del Mar, Chile.

\section{RESUMEN}

La perra doméstica se caracteriza por tener un ciclo estral, con un largo período de actividad progestagénica (diestro), situación que la predispone al desarrollo de patologías uterinas inflamatorias o del crecimiento y reparación del endometrio. La hiperplasia endometrial pseudo-placentacional corresponde a una anormalidad del crecimiento y reparación del endometrio, caracterizada por un marcado crecimiento endometrial, con patrones similares a la reacción decidual de la placentación endoteliocorial zonal, característica de la especie canina. Dada la alta actividad endometrial, clínicamente esta condición puede caracterizarse por una acumulación de contenido mucoso, sanguinolento o mucosanguinolento en el útero $\mathrm{y}$, además, puede producir que la especie canina presente una alta asociación con la inflamación endometrial, siendo predisponente para la presentación de piometra.

Palabras claves.

Perra, útero, hiperplasia endometrial pseudo-placentacional.

\section{ABSTRACT}

The bitch is characterized by an estrous cycle with a long period of progestagenic activity (diestrous), a situation that predisposes her to the development of inflammatory uterine pathologies or endometrial growth and repair. Pseudo-placental endometrial hyperplasia corresponds to an abnormality of endometrial growth and repair characterized by marked endometrial growth, with patterns similar to the decidual reaction of zonal endotheliochorial placentation characteristic of the canine species. Given the high endometrial activity, clinically, this condition can be characterized by accumulation of mucous, bloody or mucosanginolent content in the uterus and also presents high association with endometrial inflammation, being predisposing to the presentation of pyometra.

Key words.

Bitch, uterus, pseudoplacentational endometrial hyperplasia.

\section{Cómo citar:}

Sánchez Riquelme, A.E. (2021). Hiperplasia endometrial pseudo-placentacional en la perra: una revisión sucinta. Revista Facultad de Ciencias Agropecuarias -FAGROPEC. Universidad de la Amazonia. Vol. 13 (2), 169-173. https://doi.org/10.47847/fagropec. 


\section{INTRODUCCIÓN}

Entre los animales domésticos, la perra (Canis lupus familiaris) posee una biología reproductiva excepcional, clasificándosela como hembra monoéstrica típica, polítoca, no estacional y ovuladora espontánea (Concannon, 2011); esta presenta además un intervalo interestral (IIE) de entre 4 y 12 meses (Sánchez, 1999). El ciclo reproductivo de la perra se caracteriza por un prolongado período de anestro, alrededor de cuatro meses, durante el cual la hembra no evidencia signos de actividad ovárica. A esta fase le sigue un período de importante actividad folicular y de secreción de $17 \beta$-estradiol, el cual está caracterizado por la aparición de signos clínicos, tales como edema vulvar, descarga vaginal sanguinolenta y atracción del macho; este período del ciclo estral se denomina proestro y tiene una duración promedio de nueve días (Concannon, 2011).

Endocrinológicamente el proestro finaliza con la aparición del pico de hormona luteinizante (LH) (Concannon, 2011). En la siguiente fase, conocida como el estro, aparece la receptividad sexual y una marcada queratinización del epitelio vaginal; su duración promedio es de nueve días. Durante el primer tercio del estro suele ocurrir el pico de LH y la ovulación, con el consecuente desarrollo de los cuerpos hemorrágicos y cuerpos lúteos (Groppetti et al, 2015). Una vez finalizada la receptividad sexual y, en la medida en la que el epitelio vaginal disminuye significativamente - nivel de queratinización celular-, se entiende que ha comenzado el metaestro o diestro (fase lútea), durante la cual los niveles plasmáticos de progesterona se mantienen elevados (>2 ng/ml), por alrededor de 60 a 90 días (Concannon, 2011).

Durante del ciclo estral, el endometrio experimenta una serie de modificaciones macroscópicas, microscópicas y moleculares; dichos cambios están asociados, principalmente, a la influencia de estrógenos y progesterona (De Bosschere et al, 2002) (Schlafer, 2012). En la fase estrogénica ocurre una proliferación celular y un incremento de la vascularización, mientras que, en la fase progestacional, el endometrio presenta dos etapas principales de crecimiento y otra de diferenciación; luego, desde el diestro medio hasta el anestro temprano, es característica la presencia de eventos degenerativos y regenerativos (Groppetti et al, 2010).

A su vez, durante el diestro, por efecto de la progesterona, el endometrio tiene sus glándulas plenamente desarrolladas y activas. Esta condición predispone a la especie canina, al desarrollo de hiperplasia endometrial quística, a endometritis y/o a hiperplasia endometrial pseudoplacentacional (Schlafer y Miller, 2007). En este sentido, cabe destacar que, no obstante existir abundante investigación en torno a la enfermedad endometrial canina, no existe plena claridad en cuanto a la etiopatogenia de un grupo de patologías concomitantes, como sería la relación sistemáticamente propuesta entre hiperplasia endometrial quística y piometra, utilizándose, por tanto, un lenguaje técnico que tiende a uniformar dichas patologías en la forma de un complejo. Ahora bien, para ambas entidades, el rol de la progesterona en tanto generadora de cambios celulares y moleculares, en el epitelio uterino, resulta indiscutible 
(Sánchez y Arias, 2017).

Por su parte, según Schlafer (2012), las lesiones más comunes del útero canino se pueden clasificar en dos categorías: a) infecciones asociadas con endometritis; b) anormalidades del crecimiento y reparación del endometrio. Respecto de estas últimas se destacan la hiperplasia endometrial quística, los pólipos endometriales, la adenomiosis, los quistes de inclusión serosos, la subinvolución de sitios placentarios y la hiperplasia endometrial pseudoplacentacional (Schlafer y Miller, 2007). Cabe destacar que, para cuadros de hiperplasia endometrial quística e hiperplasia endometrial pseudo-placentacional, se describe que la presencia de secreciones uterinas, eventualmente podrían favorecer el establecimiento de una infección bacteriana, con endometritis (Schlafer, 2012).

\section{Hiperplasia endometrial pseudo-placentacional}

La hiperplasia endometrial pseudo-placentacional se caracteriza por una lesión uterina, con presencia de un marcado crecimiento endometrial, de características similares a la reacción decidual de la placentación endoteliocorial zonal, la que a su vez es característica de la especie canina (Nomura y Funahashi, 1999) (Schlafer y Gifford, 2008).

Siguiendo esta línea, se destaca un marcado crecimiento exuberante del endometrio, en un patrón difuso o segmentario, con alta semejanza a los tejidos maternos que se encuentran debajo de la placenta zonal canina normal. A menudo, se produce una necrosis coagulativa de los segmentos luminales de los pliegues endometriales, lo que a su vez provoca la aparición de restos amorfos en el lumen uterino, los cuales pueden confundirse con un exudado purulento (Schlafer y Gifford, 2008). En tal sentido, existe evidencia que deja ver que, la irritación leve, posiblemente asociada con una infección de bajo grado, es suficiente para iniciar esta cadena de eventos proliferativos, pero sólo cuando el endometrio está bajo la influencia de progestágenos (Chen et al, 2001).

Además, Mir et al. (2013) describen a la hiperplasia endometrial pseudo-placentacional, como una remodelación hiperplásica del endometrio, con una moderada endometritis linfoplasmática. Recientemente, Santana et al. (2020) describieron una asociación significativa, entre la frecuencia de hiperplasia endometrial pseudo-placentacional y la fase de diestro, del ciclo estral de la perra; así también lo hicieron, respecto de la relación entre hiperplasia endometrial pseudo-placentacional e inflamación uterina. Además de lo anterior, cabe señalar que la remodelación hiperplásica del endometrio, de tipo quístico o pseudoplacentacional y la endometritis subclínica, parecen tener una relación importante, con respecto de la infertilidad de las perras (Mir et al, 2013).

Igualmente, desde una perspectiva clínica, es posible señalar que las descargas vaginales suelen ser indicadores de una patología uterina, en presencia de un cuello uterino abierto (England, 2019); a su vez, según Pretzer (2008), la hiperplasia endometrial quística puede 
implicar el hallazgo de piometra, de hematometra, de hidrometra o de mucometra, destacando además, que las últimas tres entidades difieren de la piometra, en la cual, el fluido uterino es clásicamente estéril, siendo sanguinolento, seroso y seromucoso respectivamente. Así entonces, en casos de hiperplasia endometrial pseudo-placentacional se ha descrito presencia de distención uterina, con colecta de mucus (Mir et al, 2013); por lo tanto, se entiende que la presencia de secreción sanguinolenta o muco-sanguinolenta, en perras en diestro, se podría asociar con la hiperplasia endometrial pseudo-placentacional (Sánchez, 2015).

\section{DISCUSIÓN}

La hiperplasia endometrial pseudo-placentacional en la perra corresponde a una anormalidad del crecimiento y de la reparación del endometrio; esta, se caracteriza por un marcado crecimiento endometrial, con patrones similares a la reacción decidual de la placentación endoteliocorial zonal, la cual es característica de la especie canina. Dada la alta actividad endometrial, clínicamente esta condición puede caracterizarse por la acumulación de contenido mucoso, sanguinolento o mucosanguinolento en el útero; además de ello, presenta una alta asociación con la inflamación endometrial, siendo predisponente para la presentación de piometra.

\section{CONCLUSIÓN}

La hiperplasia endometrial pseudo-placentacional en perras es una entidad patológica, que afecta el crecimiento y la reparación de la mucosa uterina, lo que a su vez puede afectar la fertilidad de aquella y generar condiciones clínicas compatibles con piometra.

\section{REFERENCIAS BIBLIOGRÁFICAS}

Chen, Y., Wright, P., Lee, C. (2001). A model for the study of cystic endometrial hyperplasia in bitches. Journal of Reproduction and Fertility Suppl, 57, 407-414.

Concannon, P. (2011). Reproductive cycles of the domestic bitch. Animal Reproduction Science, 124, 200-210.

De Bosschere, H., Ducatelle, R., Vermeirsch, H., Simoens, P. \& Coryn, M. (2002). Estrogenand progesterone receptor expression in cystic endometrial hyperplasia and pyometra in the bitch. Animal Reproduction Science, 70, 251-259.

Groppetti, D., Pecile, A., Arrighi, S., Di Giancamillo A., Cremonesi, F. (2010). Endometrial cytology and computerized morphometric analysis of epihitelial nuclei: a usefull tool for reproductive diagnosis in the bitch. Theriogenology, 73, 927-941.

Groppetti, D., Aralla, M., Bronzo, V., Bosi, G., Pecile, A. \& Arrighi, A. (2015). Periovulatory 
time in the bitch: What's new to know? Comparison between ovarian histology and clinical features. Animal Reproduction Science, 152, 108-116.

Mir, F., Fontaine, E., Albaric, O., Greer, M., Vannier, F., Schlafer, D. \& Fontbonne, A. (2013). Findings in uterine biopsies obtained by laparotomy from bitches with unexplained infertility or pregnancy loss: An observational study. Theriogenology, 79, 312-322.

Nomura, K., Funahashi, H. (1999). Histological characteristics of canine deciduoma induced by intrauterine inoculation of E. coli suspension. Journal of Veterinary Medical Science, 61(4), 433-438.

Sánchez, A. (1999). Factores que afectan el intervalo interestral en la perra: una revisión. Revista Científica FCV-LUZ, 9(6), 532-536.

Sánchez, A. (2015). Hematometra e Hiperplasia Endometrial Quística en una Perra: descripción de un caso. Revista de Investigaciones Veterinarias del Perú, 26(1), 146-151.

Sánchez, A. \& Arias, F. (2017). Fundamentos y consideraciones de la patología endometrial canina. Revista de Investigaciones Veterinarias del Perú, 28(1), 1-12.

Santana, C., Santos, D., Trindade, L., Moreira, L., Paixao, T. \& Santos, R. (2020). Association of Pseudoplacentational Endometrial Hyperplasia and Pyometra in Dogs. Journal of Comparative Pathology, 180, 79-85.

Schlafer, D., \& Miller, R. (2007). Female genital system. In Maxie, M.G. (Ed.), Pathology of Domestic Animals. (pp. 429-564). Saunders.

Schlafer, D., Gifford, A. (2008). Cystic endometrial hyperplasia, pseudo-placentational endometrial hyperplasia and other cystic conditions of the canine and feline uterus. Theriogenology, 70, 349-358.

Schlafer, D. (2012). Diseases of the Canine Uterus. Reproduction in Domestic Animal, 47(Suppl. 6), 318-322. 